\title{
Inextensible Flows of Tangent Developable Surfaces of Biharmonic Curves in
}

\author{
$\mathrm{SL}_{2}(\mathrm{R})$ \\ T. Körpinar and E. Turhan*

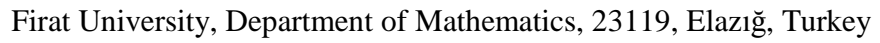 \\ Received 5 December 2011, accepted in final revised form 19 April 2012
}

\begin{abstract}
We present some results on the inextensible flows of tangent developable surfaces of biharmonic curves in the $\mathrm{SL}_{2}^{\square}(\mathrm{R})$. Finally, we find out explicit parametric equations tangent developable surfaces of biharmonic curves in the $\mathrm{SL}_{2}^{\square}(R)$.

Keywords: Biharmonic curve; $\mathrm{SL}_{2}^{\square}(\mathrm{R})$; Inextensible flows.

(c) 2012 JSR Publications. ISSN: 2070-0237 (Print); 2070-0245 (Online). All rights reserved. doi: http://dx.doi.org/10.3329/jsr.v4i2.8987 J. Sci. Res. 4 (2), 365-371 (2012)
\end{abstract}

\section{Introduction}

Developable surfaces are defined as the surfaces on which the Gaussian curvature is 0 everywhere. The developable surfaces are useful since they can be made out of sheet metal or paper by rolling a flat sheet of material without stretching it. Most large-scale objects such as airplanes or ships are constructed using un-stretched sheet metals, since sheet metals are easy to model and they have good stability and vibration properties. Moreover, sheet metals provide good fluid dynamic properties. In ship or airplane design, the problems usually stem from engineering concerns and in engineering design there has been a strong interest in developable surfaces [1].

A smooth map $\phi: N \rightarrow M$ is said to be biharmonic if it is a critical point of the bienergy functional:

$$
E_{2}(\phi)=\int_{N} \frac{1}{2}|\mathrm{~T}(\phi)|^{2} d v_{h},
$$

where $\mathrm{T}(\phi):=\operatorname{tr} \nabla^{\phi} d \phi$ is the tension field of $\phi[2-11]$.

The Euler--Lagrange equation of the bienergy is given by $\mathrm{T}_{2}(\phi)=0$. Here the section $\mathrm{T}_{2}(\phi)$ is defined by [7]

\footnotetext{
*Corresponding author: essin.turhan@gmail.com
} 
$\mathrm{T}_{2}(\phi)=-\Delta_{\phi} \mathrm{T}(\phi)+\operatorname{tr} R(\mathrm{~T}(\phi), d \phi) d \phi$,

and called the bitension field of $\phi$. Non-harmonic biharmonic maps are called proper biharmonic maps.

We present some results on the inextensible flows of tangent developable surfaces of biharmonic curves in the $\mathrm{SL}_{2}(\mathrm{R})$. Finally, we find out explicit parametric equations tangent developable surfaces of biharmonic curves in the $\mathrm{SL}_{2}^{\square}(\mathrm{R})$.

2. $\mathrm{SL}_{2}^{\square}(\mathrm{R})$

We identify $\mathrm{SL}_{2}^{\square}(\mathrm{R})$ with

$$
\begin{aligned}
& \mathrm{R}_{+}^{3}=\left\{(x, y, z) \in \mathrm{R}^{3}: z>0\right\} \text { endowed with the metric } \\
& g=d s^{2}=\left(d x+\frac{d y}{z}\right)^{2}+\frac{d y^{2}+d z^{2}}{z^{2}} .
\end{aligned}
$$

The following set of left-invariant vector fields forms an orthonormal basis for $\mathrm{SL}_{2}(\mathrm{R})$

$$
\mathbf{e}_{1}=\frac{\partial}{\partial x}, \mathbf{e}_{2}=z \frac{\partial}{\partial y}-\frac{\partial}{\partial x}, \mathbf{e}_{3}=z \frac{\partial}{\partial z}
$$

The characterising properties of $g$ defined by

$$
\begin{aligned}
& g\left(\mathbf{e}_{1}, \mathbf{e}_{1}\right)=g\left(\mathbf{e}_{2}, \mathbf{e}_{2}\right)=g\left(\mathbf{e}_{3}, \mathbf{e}_{3}\right)=1, \\
& g\left(\mathbf{e}_{1}, \mathbf{e}_{2}\right)=g\left(\mathbf{e}_{2}, \mathbf{e}_{3}\right)=g\left(\mathbf{e}_{1}, \mathbf{e}_{3}\right)=0 .
\end{aligned}
$$

The Riemannian connection $\nabla$ of the metric $g$ is given by

$$
2 g\left(\nabla_{X} Y, Z\right)=X g(Y, Z)+Y g(Z, X)-Z g(X, Y)-g(X,[Y, Z])-g(Y,[X, Z])+g(Z,[X, Y]),
$$

which is known as Koszul's formula.

Using the Koszul's formula [10], we obtain

$$
\begin{aligned}
& \nabla_{\mathbf{e}_{2}} \mathbf{e}_{1}=\frac{1}{2} \mathbf{e}_{3}, \quad \nabla_{\mathbf{e}_{2}} \mathbf{e}_{2}=\mathbf{e}_{3}, \nabla_{\mathbf{e}_{2}} \mathbf{e}_{3}=-\frac{1}{2} \mathbf{e}_{1}-\mathbf{e}_{2}, \\
& \nabla_{\mathbf{e}_{3}} \mathbf{e}_{1}=-\frac{1}{2} \mathbf{e}_{2}, \quad \nabla_{\mathbf{e}_{3}} \mathbf{e}_{2}=\frac{1}{2} \mathbf{e}_{1}, \quad \nabla_{\mathbf{e}_{3}} \mathbf{e}_{3}=0 .
\end{aligned}
$$

Moreover we put

$$
R_{i j k}=R\left(\mathbf{e}_{i}, \mathbf{e}_{j}\right) \mathbf{e}_{k}, R_{i j k l}=R\left(\mathbf{e}_{i}, \mathbf{e}_{j}, \mathbf{e}_{k}, \mathbf{e}_{l}\right)
$$


where the indices $i, j, k$ and $l$ take the values 1,2 and 3

$$
R_{1212}=R_{1313}=\frac{1}{4}, R_{2323}=-\frac{7}{4} \text {. }
$$

\section{Biharmonic Curves in $\mathrm{SL}_{2}^{\square}(\mathrm{R})$}

From (1.1) biharmonic equation for the curve $\gamma$ reduces to

$$
\nabla_{\mathbf{T}}^{3} \mathbf{T}-R\left(\mathbf{T}, \nabla_{\mathbf{T}} \mathbf{T}\right) \mathbf{T}=0,
$$

that is, $\gamma$ is called a biharmonic curve if it is a solution of Eq. (3.1).

Let us consider biharmonicity of curves in $\mathrm{SL}_{2}^{\square}(\mathrm{R})$. Let $\{\mathbf{T}, \mathbf{N}, \mathbf{B}\}$ be the Frenet frame field along $\gamma$. Then, the Frenet frame satisfies the following Frenet--Serret equations:

$$
\begin{aligned}
& \nabla_{\mathbf{T}} \mathbf{T}=\kappa \mathbf{N}, \\
& \nabla_{\mathbf{T}} \mathbf{N}=-\boldsymbol{\kappa} \mathbf{T}+\tau \mathbf{B}, \\
& \nabla_{\mathbf{T}} \mathbf{B}=-\tau \mathbf{N},
\end{aligned}
$$

where $\kappa=|\mathbf{T}(\gamma)|=\left|\nabla_{\mathbf{T}} \mathbf{T}\right|$ is the curvature of $\gamma$ and $\tau$ its torsion and

$$
\begin{aligned}
& g(\mathbf{T}, \mathbf{T})=1, g(\mathbf{N}, \mathbf{N})=1, g(\mathbf{B}, \mathbf{B})=1, \\
& g(\mathbf{T}, \mathbf{N})=g(\mathbf{T}, \mathbf{B})=g(\mathbf{N}, \mathbf{B})=0 .
\end{aligned}
$$

With respect to the orthonormal basis $\left\{\mathbf{e}_{1}, \mathbf{e}_{2}, \mathbf{e}_{3}\right\}$, we can write

$$
\begin{aligned}
& \mathbf{T}=T_{1} \mathbf{e}_{1}+T_{2} \mathbf{e}_{2}+T_{3} \mathbf{e}_{3}, \\
& \mathbf{N}=N_{1} \mathbf{e}_{1}+N_{2} \mathbf{e}_{2}+N_{3} \mathbf{e}_{3}, \\
& \mathbf{B}=\mathbf{T} \times \mathbf{N}=B_{1} \mathbf{e}_{1}+B_{2} \mathbf{e}_{2}+B_{3} \mathbf{e}_{3} .
\end{aligned}
$$

Theorem 3.1. $\gamma: I \rightarrow \mathrm{SL}_{2}(\mathrm{R})$ is a biharmonic curve if and only if

$$
\begin{aligned}
& \kappa=\text { constant } \neq 0, \\
& \kappa^{2}+\tau^{2}=-\frac{1}{4}+\frac{15}{4} B_{1}^{2}, \\
& \tau^{\prime}=2 N_{1} B_{1} .
\end{aligned}
$$

Theorem 3.2. ([9]) Let $\gamma: I \rightarrow \mathrm{SL}_{2}^{\square}(\mathrm{R}) \quad$ be a unit speed non-geodesic biharmonic helix. Then, the parametric equations of $\gamma$ are

$$
\begin{aligned}
& x(s)=\frac{1}{\aleph} \sin \varphi \sin [\aleph s+C]+\frac{1}{\aleph} \sin \varphi \cos [\aleph s+C]+\wp_{2}, \\
& y(s)=\frac{1}{\aleph^{2}+\cos ^{2} \varphi} \sin \varphi \wp_{1} e^{\cos \varphi s}(-\aleph \cos [\aleph s+C]+\cos \varphi \sin [\aleph s+C]),
\end{aligned}
$$


$z(s)=\wp_{1} e^{\cos \varphi s}$,

where $\aleph, C, \wp_{1}, \wp_{2}$ are constants of integration.

\section{Inextensible flows of Tangent Developable Surfaces of Biharmonic Curves in}

$\mathrm{SL}_{2}(\mathrm{R})$

The purpose of this section is to study tangent developable surfaces of biharmonic curves in $\mathrm{SL}_{2}^{\square}(\mathrm{R})$.

The tangent developable surface of $\gamma$ is a ruled surface [12]

$$
\mathrm{T}(s, u)=\gamma(s)+u \gamma^{\prime}(s)
$$

Definition 4.1. A surface evolution $\mathrm{T}(s, u, t)$ and its flow $\frac{\partial \mathrm{T}}{\partial t}$ are said to be inextensible if its first fundamental form $\{\mathbf{E}, \mathbf{F}, \mathbf{G}\}$ satisfies [12]

$$
\frac{\partial \mathbf{E}}{\partial t}=\frac{\partial \mathbf{F}}{\partial t}=\frac{\partial \mathbf{G}}{\partial t}=0
$$

This definition states that the surface $\mathrm{T}(s, u, t)$ is, for all time $t$, the isometric image of the original surface $\mathrm{T}\left(s, u, t_{0}\right)$ defined at some initial time $t_{0}$. For a developable surface, $\mathrm{T}(s, u, t)$ can be physically pictured as the parametrization of a waving flag.

Definition 4.2. We can define the following one-parameter family of developable ruled surface

$$
\mathrm{T}(s, u, t)=\gamma(s, t)+u \gamma^{\prime}(s, t) .
$$

Hence, we have the following theorem.

Theorem 4.3. Let $\mathrm{T}$ is the tangent developable surface associated with unit speed nongeodesic biharmonic curve in $\mathrm{SL}_{2}^{\square}(\mathrm{R})$, then $\frac{\partial \mathrm{T}}{\partial t}$ is inextensible if and only if

$$
\begin{aligned}
& -\frac{\partial}{\partial t}[\sin \varphi \cos [\aleph s+C]-u \aleph \sin \varphi \sin [\aleph s+C]]^{2} \\
& =\frac{\partial}{\partial t}[\sin \varphi \sin [\aleph s+C]+u(\aleph \sin \varphi \cos [\aleph s+C] \\
& \left.\left.-\sin ^{2} \varphi \cos [\aleph s+C] \sin [\aleph s+C]-\cos \varphi \sin \varphi \cos [\aleph s+C]\right)\right]^{2} \\
& +\frac{\partial}{\partial t}\left[\cos \varphi+u\left(\sin ^{2} \varphi \cos [\aleph s+C] \sin [\aleph s+C]+\sin ^{2} \varphi \sin ^{2}[\aleph s+C]\right)\right]^{2}, \\
& \text { where } \varphi \text { is function of time. }
\end{aligned}
$$


Proof. Assume that $\mathrm{T}(s, u, t)$ be a one-parameter family of ruled surface.

From our assumption, we get

$\mathbf{T}=\sin \varphi \cos [\aleph s+C] \mathbf{e}_{1}+\sin \varphi \sin [\aleph s+C] \mathbf{e}_{2}+\cos \varphi \mathbf{e}_{3}$.

Using first equation of the system (3.2) and (2.3), we have

$\nabla_{\mathbf{T}} \mathbf{T}=\left(T_{1}^{\prime}\right) \mathbf{e}_{1}+\left(T_{2}^{\prime}-T_{1} T_{2}-T_{1} T_{3}\right) \mathbf{e}_{2}+\left(T_{3}^{\prime}+T_{1} T_{2}+T_{2}^{2}\right) \mathbf{e}_{3}$.

By the use of Frenet formulas and above equation, we get

$\mathbf{N}=-\frac{\aleph}{\kappa} \sin \sin [\aleph s+C] \mathbf{e}_{1}$

$+\frac{1}{\kappa}\left(\aleph \sin \varphi \cos [\aleph s+C]-\sin ^{2} \varphi \cos [\aleph s+C] \sin [\aleph s+C]\right.$

$-\cos \varphi \sin \varphi \cos [\aleph s+C]) \mathbf{e}_{2}$

$+\frac{1}{\kappa}\left(\sin ^{2} \varphi \cos [\aleph s+C] \sin [\aleph s+C]+\sin ^{2} \varphi \sin ^{2}[\aleph s+C]\right) \mathbf{e}_{3}$.

Furthermore, we have the natural frame $\left\{\mathrm{T}_{s}, \mathrm{~T}_{u}\right\}$ given by

$\mathrm{T}_{s}=[\sin \varphi \cos [\aleph s+C]-u \aleph \sin \sin [\aleph s+C]] \mathbf{e}_{1}$

$+\left[\sin \varphi \sin [\aleph s+C]+u\left(\aleph \sin \varphi \cos [\aleph s+C]-\sin ^{2} \varphi \cos [\aleph s+C] \sin [\aleph s+C]\right.\right.$

$-\cos \varphi \sin \varphi \cos [\aleph s+C])] \mathbf{e}_{2}$

$+\left[\cos \varphi+u\left(\sin ^{2} \varphi \cos [\aleph s+C] \sin [\aleph s+C]+\sin ^{2} \varphi \sin ^{2}[\aleph s+C]\right)\right] \mathbf{e}_{3}$,

and

$\mathrm{T}_{u}=\sin \varphi \cos [\aleph s+C] \mathbf{e}_{1}+\sin \varphi \sin [\aleph s+C] \mathbf{e}_{2}+\cos \varphi \mathbf{e}_{3}$.

The components of the first fundamental form are

$$
\begin{aligned}
& \mathbf{E}=g\left(\mathrm{~T}_{s}, \mathrm{~T}_{s}\right)=[\sin \varphi \cos [\aleph s+C]-u \aleph \sin \varphi \sin [\aleph s+C]]^{2} \\
& +\left[\sin \varphi \sin [\aleph s+C]+u\left(\aleph \sin \varphi \cos [\aleph s+C]-\sin ^{2} \varphi \cos [\aleph s+C] \sin [\aleph s+C]\right.\right. \\
& -\cos \varphi \sin \varphi \cos [\aleph s+C])]^{2}+\left[\cos \varphi+u\left(\sin ^{2} \varphi \cos [\aleph s+C] \sin [\aleph s+C]\right.\right. \\
& \left.\left.+\sin ^{2} \varphi \sin ^{2}[\aleph s+C]\right)\right]^{2} . \\
& \mathbf{F}=g\left(\mathrm{~T}_{s}, \mathrm{~T}_{u}\right)=1, \quad \mathbf{G}=g\left(\mathrm{~T}_{u}, \mathrm{~T}_{u}\right)=1 .
\end{aligned}
$$

Using second and third equations of the above system, (4.1), (4.2), we have 
$\frac{\partial \mathbf{F}}{\partial t}=0, \quad \frac{\partial \mathbf{G}}{\partial t}=0$

Hence, $\frac{\partial T}{\partial t}$ is inextensible if and only if (4.4) is satisfied. This concludes the proof of theorem.

Corollary 4.4. Let $\gamma: I \rightarrow \mathrm{SL}_{2}^{\square}(\mathrm{R})$ is a unit speed non-geodesic biharmonic curve in $\mathrm{SL}_{2}(\mathrm{R})$. Then, the parametric equations of tangent developable surface of $\gamma$ are

$x_{\mathrm{T}}(s, u)=\frac{1}{\aleph} \sin \varphi \sin [\aleph s+C]+\frac{1}{\aleph} \sin \varphi \cos [\aleph s+C]$

$+u \sin \varphi \cos [\aleph s+C]-u \sin \varphi \sin [\aleph s+C]+\wp_{2}$,

$y_{\top}(s, u)=\frac{1}{\aleph^{2}+\cos ^{2} \varphi} \sin \varphi \wp_{1} e^{\cos \varphi s}(-\aleph \cos [\aleph s+C]+\cos \varphi \sin [\aleph s+C])$

$+u \wp_{1} e^{\cos \varphi s} \sin \varphi \sin [\aleph s+C]$,

$Z_{\mathrm{T}}(s, u)=\wp_{1} e^{\cos \varphi s}+u \wp_{1} e^{\cos \varphi s} \cos \varphi$,

where $\aleph, C, \wp_{1}, \wp_{2}$ are constants of integration.

We may use Mathematica in Corollary 4.4, which yields

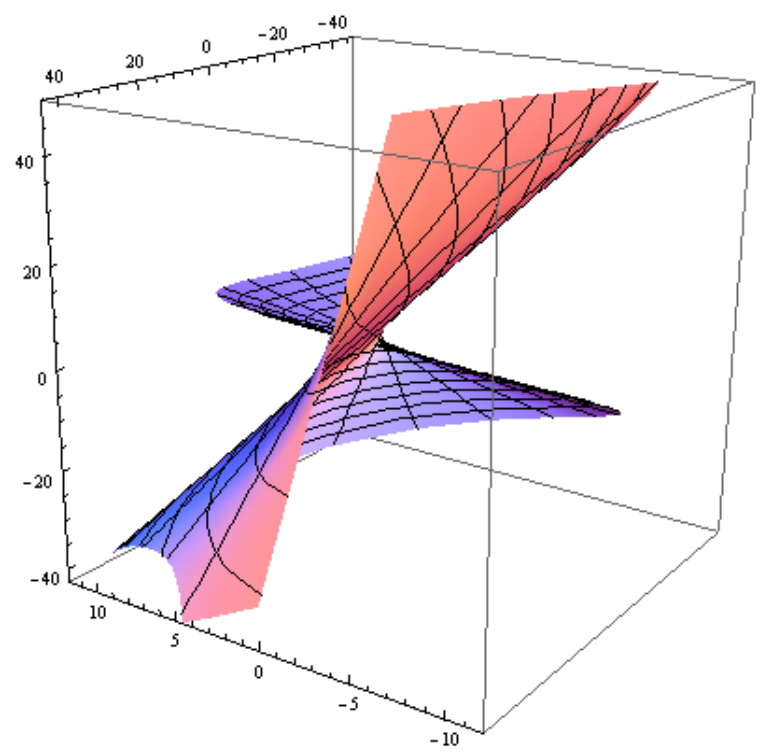




\section{References}

1. B. O'Neill, Semi-Riemannian Geometry (Academic Press, New York, 1983).

2. R. Caddeo and S. Montaldo, Intern. J. Math. 12 (8), 867 (2001). http://dx.doi.org/10.1142/S0129167X01001027

3. B. Y. Chen, Soochow J. Math. 17, 169 (1991).

4. I. Dimitric, Bull. Inst. Math. Acad. Sinica 20, 53 (1992).

5. J. Eells and L. Lemaire, Bull. London Math. Soc. 10, 1 (1978). http://dx.doi.org/10.1112/blms/10.1.1

6. J. Eells and J. H. Sampson, Amer. J. Math. 86, 109 (1964). http://dx.doi.org/10.2307/2373037

7. G. Y. Jiang, Chinese Ann. Math. Ser. A 7 (2), 130 (1986).

8. G. Y. Jiang, Chinese Ann. Math. Ser. A 7 (4), 389 (1986).

9. T. Körpınar, E. Turhan and V. Asil, Bol. Soc. Paran. Mat.- accepted (2012). www.spm.uem.br/bspm

10. E. Loubeau and S. Montaldo, math.DG/0405320 v1 (2004).

11. E. Turhan and T. Körpınar, Zeitschrift für Naturforschung - Section A Journal of Physical Sciences 65a, 641 (2010).

12. D. Y. Kwon, F. C. Park, and D. P. Chi, Appl. Math. Lett. 18, 1156 (2005).

http://dx.doi.org/10.1016/j.aml.2005.02.004 